\title{
The Future of Radio is Still Digital - But Which One? Expert Perspectives and Future Scenarios for the Radio Media in 2015
}

\author{
Marko Ala-Fossi, \\ University of Tampere, Finland \\ Stephen Lax, \\ University of Leeds, UK \\ Brian O’Neill, \\ Dublin Institute of Technology, Ireland \\ Per Jauert, \\ University of Aarhus, Denmark \\ Helen Shaw, \\ Dublin City University, Ireland
}

\begin{abstract}
The future of radio is now much less obvious and clear than it appeared ten years ago. Instead of a transition from analog to digital audio broadcasting (DAB), there is a selection alternative technological options for digital audio delivery.

This article studies how 43 people in key positions related to the radio industry in four European countries and Canada view the future of radio and which delivery technologies they consider will be most successful. In addition, it analyses the motives and reasons for why certain technologies are seen as more promising. Finally, it presents different future scenarios for radio media.
\end{abstract}


The development of digital audio broadcasting (DAB) began during the late 1980s in an EU-supported Eureka 147 project, originally intended to give the European electronics industry a competitive advantage through the digitalization of radio (Rudin 2006; Lax 2003). When DAB was first introduced in 1995, there was an optimistic and perhaps even deterministic expectation that the superior new digital radio system would replace analog FM radio in a relatively short and smooth transition from analog to digital audio broadcasting (O’Leary 1993; Kozamernik 1995; Mykkänen 1995). The first set-back came in the 1990s, when the USA and Japan decided to develop separate digital radio systems to protect their own national industries (Ala-Fossi \& Stavitsky 2003) and DAB lost the chance of becoming a truly worldwide system for digital radio. However, with the support of the World DAB organization and the European Broadcasting Union (EBU), it was widely adopted in Europe and altogether in 28 countries around the world within 10 years of its introduction (World DAB 2005). But even in those countries where DAB was implemented, the adoption rate of the new radio system was low: consumers were not interested in buying new, rather expensive digital receivers as eagerly as expected and the early phase of the classic S-shaped diffusion curve (Rogers 2003) was much less steep than anticipated. Despite the promises of new features, more choice and better (CDquality) sound, the benefits of DAB radio over FM radio were not obvious to everybody (Lax 2003).

The basic design of DAB fits better together with large, nationwide broadcasting systems and it has been more successful in countries with strong public service radio organizations (Rudin 1999; Hendy 2000). However, this has not always been the case. According to our studies of the development of digital radio in Europe and Canada, the varied success of DAB has been highly dependent on different policy decisions made by national governments and broadcasters. It seems that where the government and the public service broadcaster have both 
been committed to DAB, as in the UK and Denmark, it has been possible also to pull the commercial radio sector in with economic incentives or regulation - or both. Only in these conditions has the consumer interest in DAB radio developed favourably. This is why the UK and Denmark are among the very few countries in the world which have been relatively successful in introducing DAB. On the other hand, in Finland and in Canada, at least one of the above conditions was lacking and long-standing attempts to implement DAB have turned out to be failures. In Canada, DAB is no longer considered as the only system for digital radio, while Finland was the first country in the world to totally shut down its DAB network in 2005. For the same reasons, the DAB system in its original form had not been adopted in Ireland by 2005 except for strictly experimental use. ${ }^{1}$ (Lax et al. 2008; O’Neill 2007)

Despite the early support and great hopes for international success (Rudin 2006, p.165) in practice the EU has not been very active or generous in supporting the development of DAB or digital radio (Lembke 2003). Radio, with smaller advertising markets and more complicated national structures, has never played as significant a role in EU polices as television, and issues concerning digitalization have not been an exception to this rule. It is quite illustrative that when the EU commission finally became interested in the obstacles to the development of digital radio, the study was incorporated into a larger report about the European roadblocks on the way to the information society (Corbin 2006). According to the results, most of the roadblocks of DAB radio were related to business issues, as well as to law and technology (Goldhammer \& Veer 2006; Screen Digest et al. 2007, pp.130-135).

Although the basic reasons for the slow adoption rate and limited success of DAB may have been more social, political and economic than directly technological, during the last 10 years DAB lost not only its competitive edge as the sole digital radio system available, but it was also gradually left behind in technological development, especially in audio coding and 
multimedia capability. As noted earlier, already by the late 1990s alternative technologies for digital radio ${ }^{2}$ were developed, not only in Japan ${ }^{3}$ and the USA ${ }^{4}$, but also in Europe. Because DAB was intended to be the future of FM broadcasting, another system, Digital Radio Mondial $(\mathrm{DRM})^{5}$, was originally developed for a digital switchover of AM radio broadcasting. However, in 2005 the World DAB Forum and DRM consortium set up a project to enhance DRM for the FM band also (DRM+) (Hallet 2005). After the introduction of the first audio streaming software in 1995, the Internet also became a new platform for digital audio delivery with interactive multimedia capability (Priestman 2002). In many European countries, the gradual digitalization of television has also brought digital radio services into new digital TV networks ${ }^{6}$, while at the same time in the USA a new form of digital radio has been introduced as nationwide XM and Sirius satellite radio.

Furthermore, the rapid development of mobile technologies has recently highlighted two new and quite different trends for digital radio and audio media. Firstly, in addition to normal telephone calls, new generation mobile phone technologies ${ }^{7}$ are also able to deliver digital radio and multimedia. Secondly, new digital multimedia broadcasting systems have been developed especially for mobile use $\mathrm{e}^{8}$. Internet audio is also gradually becoming more mobile, thanks both to new technology and hardware such as proliferating wireless Internet systems ${ }^{9}$ and portable mp3 players as well as new software and programming innovations like podcasting (Berry 2006). In this way, there is now a wide selection of both competing alternative and complementary technological options for digital audio media delivery (cf. Mullane 2005). Some have similarities with analog radio broadcasting systems; others are challenging the idea of radio as solely an aural medium or even the concept of broadcasting with its tradition of linear production and reception, all of which are embedded into the traditional definition of radio. 
Because of the introduction of these alternative and in most cases more efficient systems, the 10-year old DAB system with its original audio coding system (MPEG Audio Layer II) is now widely considered to be inefficient and outdated. With more efficient audio coding, it is possible to deliver higher quality sound with lower bitrates or, equally, more services in the same amount of bandwidth. In 2005, Australia became the first country in the world to decide that DAB would be implemented only when it incorporated a more efficient audio coding system (Coonan 2005) such as MPEG-4 AAC, already supported by several other digital broadcasting standards. In October 2006, the World DAB Forum decided to adopt MPEG-4 HE-AAC v2 audio codec as an additional DAB standard, called DAB+ (Ross 2006). At the same time, the World DAB Forum changed its name to the World DMB Forum. DMB stands for Digital Multimedia Broadcasting, which is originally a DAB - based system for mobile television and multimedia broadcasting. Although the official reason for changing the name was to avoid confusion and assumptions that the organization would represent only digital audio (World DMB 2006), it also indicated clearly the end of an era for the original DAB standard. There is now no reason why any country without existing DAB provision would use the original version of DAB. However, $\mathrm{DAB}+$ is not the only new version of $\mathrm{DAB}$, as there is now also a multimedia system with support for Internet Protocol (DAB-IP). (van Gool 2006, p.6; Wright 2006, p.32; Lloyd, Maclean \& Stirling 2006)

So far, radio has been a clearly defined medium with certain established social and cultural functions and distinct delivery networks. The original vision of radio's digital future was also based on an assumption that there would be a new dedicated digital platform for radio with some additional features, but the medium as a whole would basically remain the same. It is now clear that this vision was too simplified and optimistic: on the contrary, digitalization has challenged the traditions of radio. The current proliferation of audio media on different platforms 
and the multiplicity of available options for radio delivery are, from the perspective of traditional radio, confusing and disruptive. In addition, there is no universal agreement in Europe or anywhere else over how radio - or services like it - should be delivered in the digital age. On the one hand, it seems that radio as a distinct medium in its own right is in danger of fragmenting into additional services for other digital media forms and in this way will face gradual extinction; on the other hand, the infiltration of radio-like services into practically every new delivery platform can be seen as an evidence of a "virus-like" capability of transformation and proof of the vitality of polymorphic radio media (Mullane 2005; Antoine \& Cheval 2006; Berry 2006, p.147)

Thus there are good reasons to believe that the next ten years will be very important or even epochal for radio media. According to the EU, all television broadcasting in Europe should be digital by 2012 (Hedges 2005; EU 2005; IHT 2005) and although it is most unlikely that inexpensive, ubiquitous and popular FM broadcasting would disappear overnight, there have also been some discussions of future analog radio shutdown in order to free more spectrum for other use (Ala-Fossi 2005; Ala-Fossi \& Jauert 2006, p.77). In Britain, the penetration of DAB radio is relatively high, and the Office of Communications (Ofcom) has suggested that "at some point over the next 10-15 years there may come a point where the vast majority of radio listening is via digital platforms and there may be greater value for consumers in using the VHF Band II [FM] spectrum for other things, rather than simulcasting the services also available on digital.” (Ofcom 2006, 37).

Because of this, it is now important and useful to examine how the leaders in digital technologies envision the future of digital radio. This article studies how 43 experts working with or within the radio industry in four European countries and Canada view the future of radio in 2015 and which delivery technologies they consider will be most successful. In addition, it 
analyses the motives and reasons for why certain technologies are seen as more promising - or why they are rejected in certain countries. Based on these results, the article presents a range of different future scenarios for radio media.

\section{Searching for the Digital Future(s) of Radio}

Our research project to study the technological landscape and the future of radio was launched in 2005, when DAB had its 10-year anniversary. The project was based on two earlier studies within the Digital Radio Cultures in Europe research group (DRACE): a comparative study of the development of DAB radio in the UK, Ireland, Denmark and Finland (Lax et al. 2008; Ala-Fossi \& Jauert 2006) and a separate study of DAB in Canada (O’Neill 2007). The first stage of the present study was to map all the available - both existing and emerging - technologies for delivering audio (i.e. radio and radio-like services). The special characteristics as well as the potential social and economic strengths and weaknesses of these technologies were then analysed (cf. Ala-Fossi 2005) and the data were collected in a database at the intranet website of the project.

In addition to developing our own socio-economic understanding of the technological development of radio, we wanted to find out how the future of radio was seen and understood by broadcasters, technology experts and other professionals working with the radio industry. In asking our interviewees to look forwards to the next ten years of radio, we were necessarily inviting speculative responses, but the analysis of the interviews and their scenarios was intended to reveal which (if any) of the present different technological options they believed would succeed and how they understood the current situation in which numerous options appeared to be in competition with each other. 
The primary data of this study are 43 semi-structured expert interviews, which were carried out in Ireland (3), United Kingdom (13), Denmark (6), Finland (11) and Canada (10) between May 2005 and June 2006. 14 interviewees were public broadcasters, 12 commercial or private broadcasters, 6 regulators and 5 representatives of different economic or technological interest groups, 3 network or multiplex operators and 3 persons working for the media electronics industry. The interviewees were all in senior managerial positions in their own field, and in some cases were leading members of industry groups. With only two exceptions, they were all interviewed in their native language. In addition, they were all asked the same basic set of questions, which were adapted to the context of the interview. For example, the questions were not always asked with the same words, and the questions concerning only Europe were dropped off in Canadian interviews. The interview questions were designed to explore how experts perceived the future of digital radio in terms of delivery technology, socio-economic issues, displaced technologies, and medium content. Most of the interviews were recorded during a personal meeting (39), but because of time constraints, some (4) were made via e-mail or/and by phone. $^{10}$

The research method of our study is qualitative content analysis of interview material with a grounded approach. For practical reasons, each member of the research group analysed their own research interviews conducted in their native language. In addition, the understanding of the different national contexts was absolutely crucial for this analysis. The different nuances and emphases - or even opposing views between different nationalities became more understandable in relation to the choices made in the past. Without a separate preliminary study, the different characters and dimensions of the delivery technologies would have been lost behind the large number of acronyms and abbreviations. 
Our preliminary analysis and understanding of the development digital technologies is based mainly on the social shaping of technology (SST) perspective (Mackay \& Gillespie 1992; Winston 1998; MacKenzie \& Wacjman 1999). We assume that technologies are always shaped by a combination of social, political and economic forces and processes. This is why their design and preferred forms of deployment will also match better with certain social, political or economic objectives and even exclude others. While a retrospective analysis of this social shaping of technology could readily be applied also to the development of digital radio technologies, this would be beyond the remit of the present paper. Nevertheless, some of the interviewees' responses clearly reflect the consequences of the social forces underlying the development of digital radio. However, we asked our interviewees to speculate on future developments starting, as it were, from today's technology and that is why we have also applied the concepts of the diffusion of innovations theory (Rogers 2003). Despite their many fundamental differences, SST perspective and the diffusion of innovations theory are not really antithetical and they both provide useful tools for analysing the development - and also appropriation or adoption of new technologies (Lievrouw 2006, pp. 246-261).

Especially useful for our analysis has been the classification of perceived attributes of innovations, which according to the diffusion theory explain most of the variance in the rate of adoption of innovations. Relative advantage is the degree to which an innovation is presumed to be better than the earlier system or idea. Compatibility is the degree to which an innovation is seen as consistent with the existing values, past experiences and the needs of the potential users. Complexity is the degree to which an innovation is perceived as relatively difficult to understand and use. Observability is the degree to which the results and possible benefits of an innovation are visible. Trialability is the degree to which an innovation can be experimented before adoption. (Rogers 2003, pp. 219-265). For example, the low adoption of DAB, discussed briefly 
earlier, might be interpreted so that its relative advantage over FM was not considered truly significant and its compatibility with other than nationwide public broadcasting systems was rather low.

\section{Radio in 2015: Future Scenarios from National Perspectives (Q1, Q2 and Q3)}

Each and every research interview began with the same basic question: (Q1) how do you think people will receive radio content in your country in 2015? Depending on the answer, the respondent was then asked (Q2) to explain his/her opinion and to evaluate the role of terrestrial analog, terrestrial digital, satellite and Internet radio, especially if they were not mentioned as parts of radio services in the first place. In addition, all the European interviewees were asked (Q3) what will be the dominant way of delivering radio content in Europe? Because both the interviews and their analysis have drawn on primarily national perspectives, the results are presented here following the same principle.

All the UK respondents believed that conventional, terrestrial radio would remain significant in 2015, mainly because of its familiarity and portability. Most of them (8) also believed that terrestrial broadcasting in 2015 would be predominantly digital, with DAB as the most important platform, though there were likely to be additional digital platforms such as DRM. Those who did not believe explicitly that digital would dominate in ten years' time were the community broadcasters and, less definitely, smaller commercial broadcasters. Community broadcasters (2) thought that community radio should have a place on a digital platform, but that DAB might not be the most appropriate. Although only two persons (both at BBC) believed that FM would have already been shut down, most UK respondents thought that FM would be a dying platform, appropriate mainly for new or alternative forms of radio, e.g. community radio. Two interviewees (both commercial broadcasters) also thought downloading/podcasting would become significant by 2015. 
The Danish perspectives on radio in 2015 were in many ways very similar to those in the UK. Most of the respondents thought that FM radio would still exist in 2015 and even further on, but increasingly be overtaken by digital transmission, primarily DAB and/or DRM. Also in Denmark it is likely that community radio stations especially would continue to prefer to use the FM band for some time. However, some Danish respondents (2) were convinced that the Internet will become the dominant digital platform for radio within a few years - as soon as WLAN wireless coverage is extensive enough - and to which community radio and other niche channels would eventually migrate. People would then be able to receive all sorts of radio services with new kinds of multiplatform receivers. Other respondents were less convinced about the convergence of all radio media, but some believed that the definition of radio might change over time. Radio will perhaps remain primarily an aural broadcast medium only in mobile reception, while in other contexts it may also offer other services. Although radio on-demand will co-exist with broadcasting, most radio listening will be linear and, as now, will take place while doing something else.

All the Finnish respondents - except one - believed that terrestrial analog FM radio would still be on air in 2015. However, their opinions about its importance were strongly divided. Half of group thought that FM radio would still be the dominant radio platform while the rest argued that the numbers of FM stations and their listeners would be decreasing by that time. In addition, they all thought that Finland would have some form of terrestrial digital radio broadcasting. Most believed in digital radio platform multiplicity and some were convinced that radio content will be received via multiple separate digital routes including the Internet. Five respondents - including all YLE employees - thought that digital radio delivery in the DVB-T network will continue. However, most (9) respondents considered DVB-H as the most likely system for mobile and handheld digital radio, while DMB was mentioned only as a less likely 
option. None of the respondents supported the idea that DAB would be used as a digital radio system in Finland in the future. These opinions obviously reflect the idea that the shutdown of the Finnish DAB network in August 2005 was considered to be more or less irreversible (Ala-Fossi \& Jauert 2006).

All the Irish respondents agreed that terrestrial analog FM would still be popular in 2015 and that there would be some form of digital audio broadcasting in Ireland as well. However, the full range of views on the place of digital radio was evident. The respondent representing public service broadcasting thought that DAB or DRM would be strong, perhaps the primary platform of all. The regulator thought there would still be significant FM radio, while the commercial broadcasting respondent suggested there would be very little digital listening at all.

Some Canadian respondents (3) were somewhat reluctant to predict future reception platforms for radio and insisted that informative local content would provide the most important continuity. One respondent suggested that analog broadcasting would become marginal in 10 years, but most (6) were more conservative and believed that analog terrestrial radio would remain pre-eminent in 2015. There was an understanding that digital radio would have an important place though much less certainty about what form this would take. Public service broadcasters tended to have little faith in DAB and expressed greater interest in satellite and Internet radio. Most of the commercial broadcasters thought that subscription-based satellite radio would become important though not dominant in Canada. They had mixed views on terrestrial digital radio: some believed that DAB (with DRM and DMB) could become important while others thought that it was already a failure, and would remain marginal. IBOC was widely considered as a relatively weak technology with little added value, though some respondents believed that it could become a platform for radio in Canada, especially if it was able to succeed in the US. ${ }^{11}$ 
There were, according to respondents in the study, many reasons to believe in the longevity of terrestrial analog FM broadcasting. Perhaps the most obvious is the sheer number of existing analog receivers - and their ubiquity as integrated units in all sorts of devices. Not only are FM receivers and FM transmitters relatively cheap to buy and easy to use, but both the reception and audio quality in the service area are usually good enough for the majority of users. All this makes FM especially suitable for small-scale and local radio operations. Moreover, FM radio does not have the same external pressure for digitalization that analog TV has, because the international mobile telecommunications business has been more interested in frequencies vacated by the shutdown of analog television than FM band frequencies. In some countries, (Ireland, Canada) the decline of AM radio may even strengthen FM radio. Some respondents also argued that no existing digital broadcasting system could directly substitute for FM and at the same time provide significant benefits for the radio industry or the listeners. In other words, none of the available digital systems alone has a significant relative advantage over FM which is a proven, well known and relatively simple and cheap technology that is highly compatible with the existing systems and socio-economic structures of radio broadcasting.

In general, the European respondents thought that digital radio broadcasting in their home countries would be primarily - if not solely - terrestrial. Usually, the future was seen as an extension of the present situation (cf. Wright 2005, 92), so that most of the existing systems were expected to remain in place in 2015. However, it was interesting that even in the UK and Denmark with heavy investments in DAB, many respondents were quite convinced that there is also a need for an additional digital radio system like DRM. Based on the responses, one of the main reasons for this would be the low compatibility of existing DAB system with local and community radio (Hallet 2005). In addition, DVB-T was seen as an option for the future only in Denmark and Finland. Finland was also an otherwise quite unique case, not only because there 
was so little faith in DAB - as well as in DRM - but also because very few people elsewhere saw DVB-H as a significant option for digital radio. The most obvious reasons for this emphasis also identified by some of the respondents- are the Finnish national technology policy and the involvement of Nokia in the development of DVB-H (Lax et al. 2008). The lack of a shared vision about digital broadcasting was also reflected in the Irish interviews, where the only system seen as an option by more than one respondent was DRM. Finally, the Canadian perspectives about the future of digital radio reflected two major factors: the failure of the national DAB rollout plan and a quite realistic acceptance of the influence that developments in the US radio market will eventually have on Canada (O’Neill 2007).

Practically all the European respondents agreed that satellite radio would not become important in their home countries - although some of them suspected that by 2015 there might be some sort of European satellite radio available. ${ }^{12}$ Europe was seen as a very difficult market for satellite radio, because the continent is divided into "tribal societies" not only by the number of national but also cultural and linguistic barriers. In addition, in Europe there are already nationwide and ad-free radio services in practically every national market (unlike the US, where satellite radio has had some success) which means that the demand for subscription satellite radio might be too low to make the necessary large investments profitable. ${ }^{13}$ There were also doubts about satellite radio reception, as reception is usually not good indoors and the elevation angle might be too low in northern Europe. A European satellite radio would probably need an extensive network of terrestrial filler transmitters and Europe-wide frequency clearance for such a network might be hard - if not impossible- to obtain. Satellite radio was thus seen as a highly complex system with very low compatibility and low relative advantage in European radio markets. In Canada, where both the US satellite radio systems - Sirius and XM - had just entered into the national market (O’Neill 2006, p.193) respondents had a different angle. Although 
nobody expected satellite radio to take over the Canadian radio market, it was seen as a proven system, which was supposed to fit into market and have an impact. The public service broadcaster CBC had already entered into a partnership with Sirius Radio, partly because the system with three geosynchronous satellites was so compatible with its needs: Sirius would provide CBC with full nationwide coverage including the northernmost parts of Canada.

Most respondents thought that Internet radio was already an important and growing form of radio delivery, especially for specialized and small scale services and in fixed domestic reception via broadband and WLAN. In the most radical visions, Internet radio would also be available “everywhere” via wireless in 2015, though some technology experts characterized the future of Internet radio at its best as "portable”, but not truly mobile (e.g. for in-car reception). As mentioned earlier, the strongest believers in the development of Internet radio were among the Danish and the Canadian respondents, while it was seen as most problematic among the Finns, mostly because of copyright issues. This difference reflects again the national contexts: unlike in the other countries, a longstanding dispute over music royalty payments has in effect frozen the development of Internet radio in Finland (Ala-Fossi \& Jauert 2006, pp. 72-73). ${ }^{14}$ In general, based on our study, it seemed that while most respondents had a comprehensive understanding of the relevant national market, they were not always fully aware of developments in other countries, and so did not have in-depth knowledge of all the available options for delivering radio (for example DRM).

No single platform or technology was raised above the others by the European respondents: there was no clear consensus about the dominant European way of delivering radio in 2015. However, some common trends were evident. First of all, only a few people altogether thought that DAB still had any chance of becoming the dominant platform in Europe. Even in the UK and Denmark some respondents argued that DAB was already an outdated system which 
would be supplemented or even replaced by DRM. (In general, DAB was seen as remaining an important platform for those countries where it already existed, but other technologies might become more popular in other European countries. Thus the majority of respondents agreed that there would be several parallel distribution platforms for radio in Europe, depending on which systems are considered appropriate in each country but also depending more generally on developments in the purposes of the radio operations and consumer preferences. While the Irish respondents did not have a common view on which was the best alternative to $\mathrm{DAB}$, the majority of the Finns saw DMB and DVB-H as the main rival options for the further digitalization of radio delivery in Europe. Finally, although the continuing existence of FM radio in Europe was seen as clearly likely by most, only very few respondents argued that FM would still be the dominant European radio delivery system in 2015.

\section{Spotting the Dinosaurs: How to Identify the Winners from Future Losers (Q4, Q5)}

After outlining their views on the future of radio, each respondent was asked (Q4) why they thought so and to explain their arguments by exploring the following perspectives: regulation, ownership, market penetration, economic issues, production practices, geographic coverage area, functionality and user practices. The research team had used the same set of eight different perspectives in its preliminary socio-economic study on the delivery technologies. However, as they were not strictly defined during the interviews, the perspectives worked here more as an inspiration than an instruction for the interviewees. In addition, the respondents were asked to (Q5) name technologies which will not be dominant in the future, but would be still in use.

There was a wide variety of opinions about the role and impact of regulation among the respondents. Some claimed that regulation had very limited effects, while others argued that 
the lack of regulation or its inappropriateness could be decisive in digital radio development. Respondents, especially from the UK and Ireland, saw national regulation in the form of incentives as an important element in the relative success of DAB in the UK. The importance of economic incentives on technological choices in commercial broadcasting was also recognized elsewhere (cf. Ala-Fossi \& Jauert 2006, p.78). On the other hand, it was widely felt that national differences in government regulation and frequency administration would contribute to a fragmentary European situation for digital radio delivery. Respondents in Finland and Canada saw their national regulation system as more "market-driven” than for example in Denmark. While most Finns considered regulation as a highly national issue also in the future, some Danes argued that national legislation would play a steadily decreasing role and that EU media regulation would have a much more central role than previously. In addition, technology policy both on the national and EU levels was also considered as a form of regulation by some respondents.

The ownership perspective was seen as important mainly by the Finnish respondents. Two different dimensions were identified: the ownership and copyright of delivery technologies themselves and the ownership and copyright of the media content delivered. Two respondents working in regulatory agencies stated that only open, non-proprietary technologies like DVB-H - could have wider success in the long run. Any tendencies towards corporate monopolization of delivery technologies would not be tolerated by the market or the governments. In terms of content, in addition to the problems with Internet radio in Finland, there was also an on-going dispute about copyright payments and music royalties for simulcasting in the DVB-H network (Ala-Fossi \& Jauert 2006, pp.72-73). This probably explains why the content ownership and copyright dimension were considered very problematic by the Finns in 
particular: some also thought that expensive content copyright payments could effectively prevent the use of certain radio delivery technologies.

The existing market penetration was widely considered as one of the major strengths of FM, but also as favourable for DAB. In those countries where a DAB infrastructure was already in place, the cost of installing a different infrastructure for an alternative technology would be prohibitive. This would also help the introduction of DMB, which could use the DAB infrastructure. On the other hand, where DAB had a high market penetration, any introduction of system updates like new audio coding would only be very gradual. However, the market penetration of DAB in the UK and across Europe in general was considered by some Irish and Finnish respondents to be rather low in absolute terms, despite the extensive marketing in those countries where it was regarded as relatively successful. Some Canadian respondents argued that if a technology achieved a high enough penetration in the larger US markets, it would mean that the same standard would later also be adopted by Canada, not least because large manufacturers will offer the same products for both markets.

Many of the problems with DAB were understood by the respondents as principally economic issues. DAB transmission was widely considered as expensive and uneconomic especially for commercial and community stations when the costs were compared to analogue transmission or to alternative digital broadcasting systems. This relative disadvantage of DAB from an economic perspective is caused more by the design of its implementation (multiplexes) and basic architecture (audio coding system) than for example by multiplex operator pricing policy. At the same time, some respondents argued that an alternative multimedia system network with more efficient audio coding would provide not only higher capacity, but it would also be more economic with better cost efficiency per bit for the content operators. New services were seen as potential sources of new income for broadcasters and multiplex operators and, for 
example, some Finnish respondents stated that DVB-H was attractive because unlike DAB it offered the opportunity to identify the users and sell both content and services also directly to them. From the economic perspective, Internet radio and satellite radio were seen by several respondents as expensive solutions. Satellite radio would need huge investments, while Internet radio required more money to cover royalties and higher delivery costs for additional listeners.

DAB was also perceived as having problems with coverage. In the UK and Denmark, it had become obvious that community radio and small commercial stations were not suited to the existing coverage patterns of DAB multiplexes (cf. Corominas et al. 2006). The Irish respondents agreed that DAB coverage would not be compatible with the needs of local radio stations in Ireland and this was one of the main reasons why DAB had not secured widespread support in that country. In Finland in particular, the larger private broadcasters were not interested in paying for coverage in the sparsely inhabited northern areas and the cost of building and running a DAB network with comprehensive coverage would also be relatively high in Canada. In addition, lack of robust DAB indoor reception was also acknowledged as a problem by interviewees both in the UK and Denmark. In contrast, FM radio was widely seen as well suited for community radio - and Internet radio was considered a good way to extend its coverage where needed. In Europe, terrestrial delivery was also thought to have significant benefits over satellite: the ability to provide local programming, the ease of producing programming for audiences in a single nation and ease of reception and consumption. Meanwhile in Canada, the extensive satellite service was seen as a good way to produce truly nationwide coverage of radio services.

Most Canadian respondents thought that user practices were important determining factors and that those adopted by younger listeners in particular would be the most successful in long run. Some also believed that in-car reception would be a more important factor in North 
America than, for example, in Japan, where more people commute by train. In comparison, fewer European respondents saw user practices as important. For example, only one person in the UK and two in Finland thought that the consumers would be key in deciding which platform would succeed. Furthermore, it was argued that DAB had the advantage that it is familiar to users as a digital form of 'conventional' radio. However, this kind of compatibility with analog radio was not necessarily intentional but was more a result of the lack of development of DAB's multimedia capabilities. For example, in Denmark DR marketed DAB by emphasizing the diversity of services instead of the new dimensions of the radio experience. Some Finnish respondents believed that the future users of digital radio would expect to have their receivers integrated into a cellphone-like, handheld personal media device. There were also totally opposite views: it was also thought unlikely that one application would cover all user practices especially if it required an expensive terminal and a subscription.

The technical functionality of a particular digital radio system was generally not seen as a very important factor. Some respondents even argued that everything else may matter more than the system's technical performance. Even very good functionality could not guarantee success, but on the other hand insufficient performance or the lack of certain characteristics - like good mobile reception- could be a burden. In the case of DAB the old audio coding and the lack of real multimedia capacity were seen as fatal by some Finns. At the same time, some respondents from the UK saw DAB as a proven system, even the only proven system, which could offer additional functionality, although this argument failed to acknowledge the recognition of DAB's poor reception in certain circumstances (e.g. indoors). As mentioned earlier, satellite radio’s indoor reception was also questioned. DRM was thought to have good functionality as a modern system with better audio coding than DAB. Of all the possible factors for the future development of radio, production practices were considered to be among the least important. 
Some respondents even argued that this would not matter at all, although they were mentioned in occasional references to podcasting and Internet radio as alternatives to broadcast radio.

Among the European respondents, DRM was the favoured option as an important but not necessarily dominant radio delivery system in the future. In the UK and Denmark there were also some who thought that DRM might not just exist alongside DAB, but perhaps even replace it. Almost all UK interviewees and some Danes also anticipated that in the near future electronic chips in radio receivers would be multiplatform (DAB/DRM/FM) ${ }^{15}$ while some Finns suggested that software radio would provide the solution to platform multiplicity (Sabel 2007). Another widely accepted as important, but non-dominant radio platform, was the Internet with all IP-based solutions like Internet radio and podcasting - and some Danish and Canadian respondents thought that the Internet would most likely become the dominant radio platform. DAB, DMB, DVB-H and mobile phone networks were also mentioned occasionally, but nobody suggested that satellite radio or IBOC would become even secondary platforms for radio delivery in Europe.

\section{Screening the Imagined Dial(s): What will be on the Radio(s) in 2015? (Q6, Q7)}

The two final questions of the interview structure were not about radio delivery or radio technologies as such, but instead the respondents were asked (Q6) what they think will be the content of radio media and what are the listeners or users going to be doing with it. If necessary, they were also asked to (Q7) explain why they thought the content of radio in 2015 would be like that. Some of the respondents argued that they were not experts in these issues, but they were willing to speculate about the future trends of radio and audio content.

In the responses, there were two main trends, evident in responses from all countries. On the one hand, radio in the future was expected to offer much more personalized and specialized content - also with multimedia elements - which would then be actively selected by 
the users for listening whenever they found it suitable. This sort of non-linear use of radio or ondemand radio would be free from the strict time schedules and the linear flow of normal broadcast radio. On the other hand, several respondents were convinced that most radio content and its use in the future would be very similar to its current form - some even suggested that, thanks to new channels, there would also be a renaissance of the most traditional genres and forms of radio content. Some respondents however were rather pessimistic about the future of traditional and information-based, real-time linear radio program services, but in most cases the mutual co-existence of these two developments was seen as possible or even very likely (cf. Scannell 2005).

Thus it was believed that traditional broadcast radio, which is a very time-based, linear medium where you have to be tuned in to certain channel at certain time and follow the schedule designed by the broadcaster to be able to get the content what you want, would become partly a thing of the past. Instead, one of the common characteristics of newly emerging digital platforms for radio was assumed to be higher listener and user sovereignty, a result both of the increasing number of channels (satellite and terrestrial digital radio as well as the Internet) and of non-linear content delivery and consumption (downloading, podcasting and listening of recorded audio files). Besides circumventing schedules, listening to downloaded, recorded programmes will also help overcome poor signal reception, especially when on the move. Some respondents emphasizing the importance of Internet argued that future media users will not accept that they cannot use radio and television in the same way that they use newspapers and the Internet - they will not wait till a certain time of day to listen to the news or to jazz, as they become used to consuming media whenever and wherever they wish. (Ofcom 2004) This was seen as a challenge to broadcasters who are used to designing their production with a certain program grid and for only one delivery channel. 
There were quite different views about the importance of multimedia aspects of digital radio delivery. Three Finnish respondents suggested that radio lacked visuality and that this would be an important reason for overall digitalization of radio content delivery, while two others thought that the most important multimedia characteristic would be direct sales of content and services to the users. Some UK respondents thought that the most likely multimedia features of digital radio would be downloading music and radio programmes for later listening, while one Irish respondent believed that digital radio’s electronic programme guide would be reasonably attractive to listeners. Some respondents with a more technical background thought also that radio would find a totally new kind of interactivity with the users, which would go beyond the current phone-in, SMS, WAP and Internet applications. Others, however, were sceptical of the benefits of digital radio multimedia and thought that the Internet or mobile phone systems would be better for downloading data than digital radio broadcasting.

It was also widely thought that the increasing number of channels available would also lead to increasing specialization of radio content - just as in satellite radio - so that there would be special, themed channels for every genre of music as well as more channels with no music at all. Some Finnish respondents thought that in the future a growing amount of radio or audio content would be produced (or re-produced) by audiences and one respondent suggested that Internet radio delivery could also be used for non-edited, linear real-time audio flow from some live events. ${ }^{16}$

Perhaps it is not surprising that, among the respondents from every country, there was a strong - although not unanimous - belief in linear broadcast radio programming with real time listening, in other words the traditional strengths of radio would be retained. Mobility, easy access, real-time broadcasts, localism and integration with the community, personalities, entertainment as well as different types of traditional journalistic and artistic audio programming 
were all considered important. This kind of broadcast flow radio consumption was described as "passive” radio listening or lean-back radio. It was argued that in 2015 there would continue to be a demand for broadly similar radio content as now and familiar radio-type services, which do not require too much from the user or the listener. In addition, most Danish respondents and many Finns thought that professionally produced radio content even in its most traditional forms will have its place in the future. However, some Danish respondents suggested that for example radio drama and documentaries have so far been severely handicapped by broadcast scheduling and their possible renaissance would be a consequence of the growth of on-demand radio. They also thought that even though the content of radio overall would be the much same as it is now, there would be differences in which types of content were linked to which platforms.

\section{Discussion: Future Scenarios for the Radio Media}

Most respondents clearly believed that some sort of digital terrestrial radio broadcasting would exist in their home countries in 2015. DAB was seen as a strong option mainly in Britain and in Denmark, but even there it was expected to have supplementary digital platforms like DRM and DMB. In Finland DVB-H was considered as the most likely option, while in Canada it was thought that there might also be IBOC alongside DAB and satellite radio. With few exceptions, most respondents believed that analog FM broadcasting would remain significant until 2015 both in Europe and Canada. There were variable views about Internetbased radio and audio services everywhere, but the idea of successful satellite radio services in Europe was rejected by most European respondents.

The European respondents did not agree on any single dominant digital audio delivery platform. On the contrary, most of them thought that there would be distinct national solutions and multiple, co-existing digital systems for radio delivery that is several different digital futures for radio instead of one, with DAB was seen as particularly important primarily in 
countries with already existing networks. Many respondents argued that the relative advantage of DAB for both the broadcasters and listeners was low - and its compatibility with the existing socio-economic structures of radio broadcasting was not satisfactory. ${ }^{17}$

The explanation offered for this is that the original basic design of DAB reflects the socio-political and economic structures of European radio broadcasting in the 1980s. At the time when DAB was created, in many European countries private and local radio broadcasting was either non-existent or about to be introduced, and radio in general was still largely dominated by national public radio broadcasters. However, over the next few years the structures, competitive settings and power relations of radio broadcasting in Europe changed in a fundamental way - but all this was more or less neglected in the development of DAB system (Vittet-Philippe \& Crookes 1986, pp.8-24; Rudin 2006, p.167). In other words, when DAB was finally introduced in 1995, it faced a very different sociopolitical environment than where it was originally designed (cf. Ala-Fossi \& Stavitsky 2003).

Here lies also the reason why DRM is now expected to complement or to replace DAB - although it has less capacity for data and sound, it does not challenge as many existing economic and social structures of radio broadcasting as DAB. Using DRM, each broadcaster can keep their own transmitters and networks and continue operations on separate frequencies, as they do currently with analogue radio, instead of joining a shared multiplex - and this also makes the DRM coverage patterns more flexible. In this way, it is much more compatible with the diversity of current forms of analog radio broadcasting in Europe than DAB. An unresolved question is whether DRM will be able to offer sufficient relative advantage (over analog FM) both for the broadcasters and for the listeners. In any case, what this proves that by the time of the interviews, the original vision of a transition of radio in Europe and across the world to one single 
superior digital system had already failed for good. It remains to be seen whether the new versions of DAB (DAB+, DAB-IP and DMB) will be more successful.

It was interesting to see that the idea of dual development of future radio content was so widely supported among the respondents in all five countries. However, this division into traditional linear broadcast audio content with real time listening and to a new, increasingly personalized type of content, which is received on-demand and consumed off-schedule, in fact already exists: web-based radio and audio services in particular have recently expanded the territory of radio media and to some extent have overcome their earlier limitations. A prediction which assumes that both types of content and consumption will also exist in the near future is hardly radical: on the contrary, it could be described as merely realistic or perhaps even a bit conservative. At the same time, there was no consensus about whether traditional, linear radio produced by broadcasters would still have a significant share in 2015 or whether the majority of users would prefer freedom from schedules and personalized content over real-time radio.

The development trends in adoption of radio delivery technologies and the development of radio content consumption are both very important and, based on our results, still highly uncertain issues. This is why they are perfect for scenario building: using these two variables, it is possible to create a scenario matrix to describe four different future scenarios for radio media. The $X$ axis indicates the options in delivery technologies, while the $Y$ axis describes the tendencies in radio and audio content consumption (Wright 2005, pp. 98-99). Because all the respondents were asked questions about both the development and adoption of delivery technologies (Q1 - Q5) and about radio content and its consumption (Q6 - Q7), it is also possible to estimate how closely they could be identified with these scenarios (see Figure 1.). The location of each respondent in the scenario matrix is defined here according to $X$ and $Y$ values between 0 
and 2 allocated by members of the research group, based on subjective evaluation of the interviewee's opinions, choices and arguments.

In order to make the matrix more understandable, it is necessary to give a brief description of each scenario. “Towers of Babel” is actually very similar to the present situation, where free analog broadcast delivery and real time consumption of live (or otherwise linear) audio content are still the most important ways to produce and to use radio. Traditional broadcast operators are still offering the majority of the services. In addition, there are services using many competing digital technologies, but none of the technologies has a really dominant position anywhere and this is why digital radio remains small. In "DAB DReaM" free broadcast delivery and real time consumption of live or linear audio content also remain the most important forms of radio. Again, traditional broadcasters deliver most of the services. However, besides strong analog radio there is fast growing digital radio with a clearly dominant technology or a selection of complementary digital technologies (e.g. DAB/DRM/DMB). “Digital Diversity” means that digital radio has a different (dominant) design in different parts of the world and there can be very distinct national solutions. In addition, in most countries there are several competing digital systems for radio delivery. In some cases, linear radio-type audio is just a small fraction of the services available. Multimedia, subscription and on-demand audio services provided by producers other than broadcasters are gradually increasing and becoming at least as important as traditional broadcast audio. In "Multimedia Market” digital radio is growing with one dominant technology or group of technologies (e.g. DVB-T/DVB-H), but the role of traditional broadcast radio operators is diminishing. Multimedia and on-demand audio is also offered by nonbroadcasters and is becoming as significant as traditional broadcast audio.

The respondents' locations on the scenario matrix appear to reflect their national contexts at least as much as professional contexts. For example, most of the people supporting 
the “DAB DReaM" scenario were those other than broadcasters from countries with existing DAB systems; broadcasters were also a small minority among the respondents supporting a future scenario like “Multimedia Market”. On the other hand, the supporters of a "Towers of Babel" scenario were mostly broadcasters while a scenario like “Digital Diversity” was most commonly accepted especially among public broadcasters.

These four scenarios, however, do not provide a fully comprehensive vision of the possible futures of radio media. The choices between the delivery technologies are more important the more exclusive they are, and these scenarios do not implicitly take into account any socially shaped development in reception systems. The present multiplicity of different delivery systems for radio has been shaped by complex social, political and economic processes. As a consequence, there is now a need for a new generation of receivers - or "end user terminals". A multiplatform receiver (e.g. AM/FM) is hardly a new invention, and the latest development of reception devices has been very promising.

In addition, a true "Software Radio" is no longer just science fiction (Sabel 2007). This sort of flexible, programmable radio receiver provides compatibility with a wide selection of delivery platforms. At least in the beginning, such receivers will be also complex and expensive devices - while the radio receiver, as we have come to know it, has been a cheap and simple to use. Whatever the outcome of that development will be, the future of radio is always dependent on what people - the listeners - want to do with the content of this media. 


\section{References}

Ala-Fossi, M. \& Jauert, P. (2006) Nordic Radio in the Digital Age. In Carlsson, U.(Ed.) Nordic Media Trends 9: Radio, TV \& Internet in the Nordic Countries. Meeting the Challenges of New Media Technology. (pp. 65-87). Göteborg: Nordicom.

Ala-Fossi, M. (2005, November) Mapping the Technological Landscape of Radio: Where do we go next? Paper for session: Digital Technologies and the Future of Radio at the First European Communication Conference, Amsterdam, The Netherlands.

Ala-Fossi, M. \& Stavitsky, A.G. (2003) Understanding IBOC: Digital Technology for Analog Economics. Journal of Radio Studies, 10, 63-79.

Antoine, F. \& Cheval, J. (2006, November) Mutation pour le premier média mondial. « Les Radios succèdent à «La Radio». IREN Communiqué. [Mutation of the first global media. "The Radios” succeed "the Radio". An official statement of IREN] Brussels, Belgium.

BBC (2007, January 26) Recycled satellite radio planned. BBC News. Retrieved February 2, 2007, from: http:/news.bbc.co.uk/2/hi/technology/6301359.stm

BBC (2006, September 28) UK to get satellite radio by 2010. BBC News. Retrieved October 1, 2006, from: http://news.bbc.co.uk/2/hi/entertainment/5389522.stm

Berry, R. (2006) Will the iPod Kill the Radio Star? Profiling Podcasting as Radio. Convergence: The International Journal of Research into New Media Technologies. 12, 143-162.

Coonan, H. (2005, October 14) Speech by Australian Minister for Communications, Information Technology and the Arts about Digital Radio. Commercial Radio Australia Conference, Sydney. Retrieved October 26, 2005, from: http://www.minister.dcita.gov.au/media/speeches/digital_radio__commercial_radio_australia_conference

Corbin, C. (2006, October 14) Information Society roadblocks identified! Retrieved December 7, 2006, from:

http://www.epsiplus.net/news/information_society_roadblocks_identified

Corominas, M.; Bonet, M.; Guimerà, J. \& Fernández, I. (2006) Digitalization and the Concept of "Local”: The Case of Radio in Spain. Journal of Radio Studies, 13, 116-128.

EU (2005, May 24) Commission expects most broadcasting in the EU to be digital by 2010.

[Press release] Retreived July 27, 2005, from: http://europa.eu.int/rapid/pressReleasesAction.do?reference=IP/05/595\&format=H TML\&aged $=0$ \&language $=$ EN\&guiLanguage $=$ en

ESA (2007, January 25) Multimedia car radio of the future. [Press release] Retrieved January 29, 
2007, from the European Space Agency website:

http://www.esa.int/esaCP/SEM9OBSMTWE_index_2.html

Goldhammer, K. \& Veer, C. (2006, July 3) Radio-DAB. Main Roadblocks. Preliminary results of a study for the European Commission Information Society Directorate Unit C1, presented in Brussels, Belgium. Retrieved December 28, 2006, from:

http://europa.eu.int/information_society/eeurope/i2010/docs/conf_wshop/workshop _slides.ppt

Hallet, L. (2005, June) DRM Expands Into FM Sphere. Radio World International Edition, 1-3.

Hedges, M. (2005, January 3) Pushing the Switch Slowly. followthemedia.com. Retrieved July 22, 2005, from: http://followthemedia.com/alldigital/switch0103.htm.prn

Hendy, D. (2000) A Political Economy of Radio in the Digital Age. Journal of Radio Studies, 7, 213-233.

IHT (2005, May 25) EU urges digital TV conversion by all states. International Herald Tribune. Retrieved September 26, 2005, from:http://www.iht.com/articles/2005/05/24/business/eudig.php

Kozamernik, F. (1995, Autumn) Digital Audio Broadcasting - radio now and for the future. EBU Technical Review, 2-27. Retrieved September 22, 2005, from: http://www.ebu.ch/en/technical/trev/trev_265-kozamernik.pdf

Lax, S. (2003) The Prospects for Digital Radio. Policy and technology for a new broadcasting system. Information, Communication \& Society 6, 326-349.

Lax, S.; Ala-Fossi, M.; Jauert, P. \& Shaw, H. (forthcoming, 2008) DAB: the future of radio? The development of digital radio in four European countries. Media, Culture \& Society, 30 (1).

Lembke, J. (2003) Strategies, Politics and High Technology in Europe. Comparative European Politics, 1, 253-275.

Lievrouw, L.A. (2006) New Media Design and Development: Diffusion of Innovations v Social Shaping of Technology. In Lievrouw, L.A. \& Livingstone, S. Handbook of New Media: Social Shaping and Social Consequences of ICTs. Student edition.(pp. 246265) London: Sage

Linstone, H.A. \& Turoff, M. (Eds.) (1975) The Delphi Method. Techniques and Applications. Massachusetts: Addison-Wesley Publishing Company. Retrieved November 23, 2005, from: http://is.njit.edu/pubs/delphibook/

Lloyd, E.; Maclean, R. \& Stirling, A. (2006, April) Mobile TV - results from the BT Movio DAB-IP pilot in London. EBU Technical Review. Retrieved June 20, 2006 from: www.ebu.ch/en/technical/trev/trev_306-movio.pdf 
Mackay, H. \& Gillespie, G. (1992) Extending the Social Shaping of Technology Approach: Ideology and Appropriation. Social Studies of Science, 22, 685-716.

MacKenzie, D. \& Wacjman, J. (Eds.) (1999) The Social Shaping of Technology. Second edition. Maidenhead and Philadelphia: Open University Press

Mullane, M. (2005) More platforms than Grand Central Station. DIFFUSION online 2005/35, EBU Radio Department. Retrieved October 15, 2006, from: http://www.ebu.ch/CMSimages/en/online_35_e_radio_news_tcm6-39591.pdf

Mykkänen, J. (1995) Yleisradiotoiminnan strategiaselvitys. Radio ja televisio 2010. [A Strategy Report on Broadcasting.Radio and Television 2010.] Liikenneministeriön julkaisuja 45/95. Helsinki: The Finnish Ministry of Transport and Communications.

O’Leary, T.(1993, Spring) Terrestrial Digital Audio Broadcasting in Europe. EBU Technical Review, 19-26. Retrieved September 22, 2005, from: http://www.ebu.ch/en/technical/trev/trev_255-o_leary.pdf

O'Neill, B. (2007) Digital Audio Broadcasting in Canada: Technology and Policy in the Transition to Digital Radio. Canadian Journal of Communication, 32, 71-90.

O’Neill, B. (2006) CBC.ca. Broadcast Sovereignty in a Digital Environment. Convergence: The International Journal of Research into New Media Technologies. 12, 179-197.

Ofcom (2006, November 16) The Future of Radio. The future of FM and AM services and the alignment of analogue and digital regulation. [Discussion document] Retrieved November 20, 2006 from: http://www.ofcom.org.uk/consult/condocs/radio_future/

Ofcom (2004, July 23) The iPod Generation. Devices and Desires of the Next Generation of Radio Listeners. Prepared for Ofcom by the Knowledge Agency. Retrieved September 15, 2004 from:

http://www.ofcom.org.uk/research/radio/reports/ipod_gen/ipod.pdf

Priestman, C. (2002) Web Radio: Radio Production for Internet Streaming. London: Focal Press

RadioScape (2006, November 20) RadioScape now shipping multi-standard DAB/DRM modules to meet early market demand. First multi-standard consumer radios now on sale. [Press release]. Retrieved November 28, 2006, from: http://www.radioscape.com/downloads/Press_Release/RS201106.pdf

Rogers, E.M. (2003) Diffusion of Innovations. (5th ed.) New York \& London: Free Press.

Ross, C.T. (2006, December) Eureka 147 Adds Option For Coding. Radio World International Edition, 1.

Rudin, R.(1999) Eureka 147: Digital Diversity or Radio Restriction? MA Dissertation in Mass 
Communications. University of Leicester, Leicester, UK. Retrieved March 20, 2002, from: http://www.richardrudin.com

Rudin, R. (2006) The Development of DAB Digital Radio in the UK. The Battle for Control of a New Technology in an Old Medium. Convergence: The International Journal of Research into New Media Technologies. 12, 163-178.

Sabel, L. (2007, January) Software-defined radio - the solution for multi-standard multimedia in the mobile environment. EBU Technical Review. Retrieved February 10, 2007, from: http://www.ebu.ch/en/technical/trev/trev_309-radioscape.pdf

Scannell, P. (2005) The Meaning of Broadcasting in the Digital Era. In Lowe, G. \& Jauert, P. (Eds.) Cultural Dilemmas in Public Service Broadcasting. RIPE@2005. (pp. 129-142) Göteborg: Nordicom.

Screen Digest et al.(2007, January 25) Interactive content and convergence: Implications for the Information Society. A study for the European Commission (DG Information Society and Media) by Screen Digest Ltd, CMS Hasche Sigle, Goldmedia Gmbh, Rightscom Ltd. London: Screen Digest Limited. Retrieved February 10, 2007, from: http://ec.europa.eu/information_society/eeurope/i2010/studies/index_en.htm

van Gool, C. (2006, December) IP-DAB Performs Well in Tests. Radio World International Edition,1.

Vittet-Philippe, P. and Crookes, P. (1986) Local Radio and Regional Development in Europe. Manchester: The European Institute for the Media.

Winston, B. (1998) Media Technology and Society. A History: From the Telegraph to the Internet. London and New York : Routledge.

World DAB (2005, April 14) New wave of DAB legislation and developments worldwide. [Press release] London: World DAB. Retrieved September 12, 2005 from:

http://www.worlddab.org

World DMB (2006, October 30) World DAB Forum changes its name. [Press release]. London: World DMB. Retrieved November 1, 2006, from: http://www.worlddab.org/upload/uploaddocs/WorldDMB_PR_301006.pdf

Wright, A. (2005) Using Scenarios to Challenge and Change Management Thinking. Total Quality Management and Business Excellence. 16, 87-103.

Wright, M.(2006, August 3) ETSI adds IP support to DAB, simplifying a DVB-H migration. EDN. Retrieved November 11, 2006 from:

http://www.edn.com/article/CA6356077.html?industryid=2282\&text=dvb\%2Dh 


\section{Figure 1}

\section{Division of the respondents between the four future scenarios for the radio media}

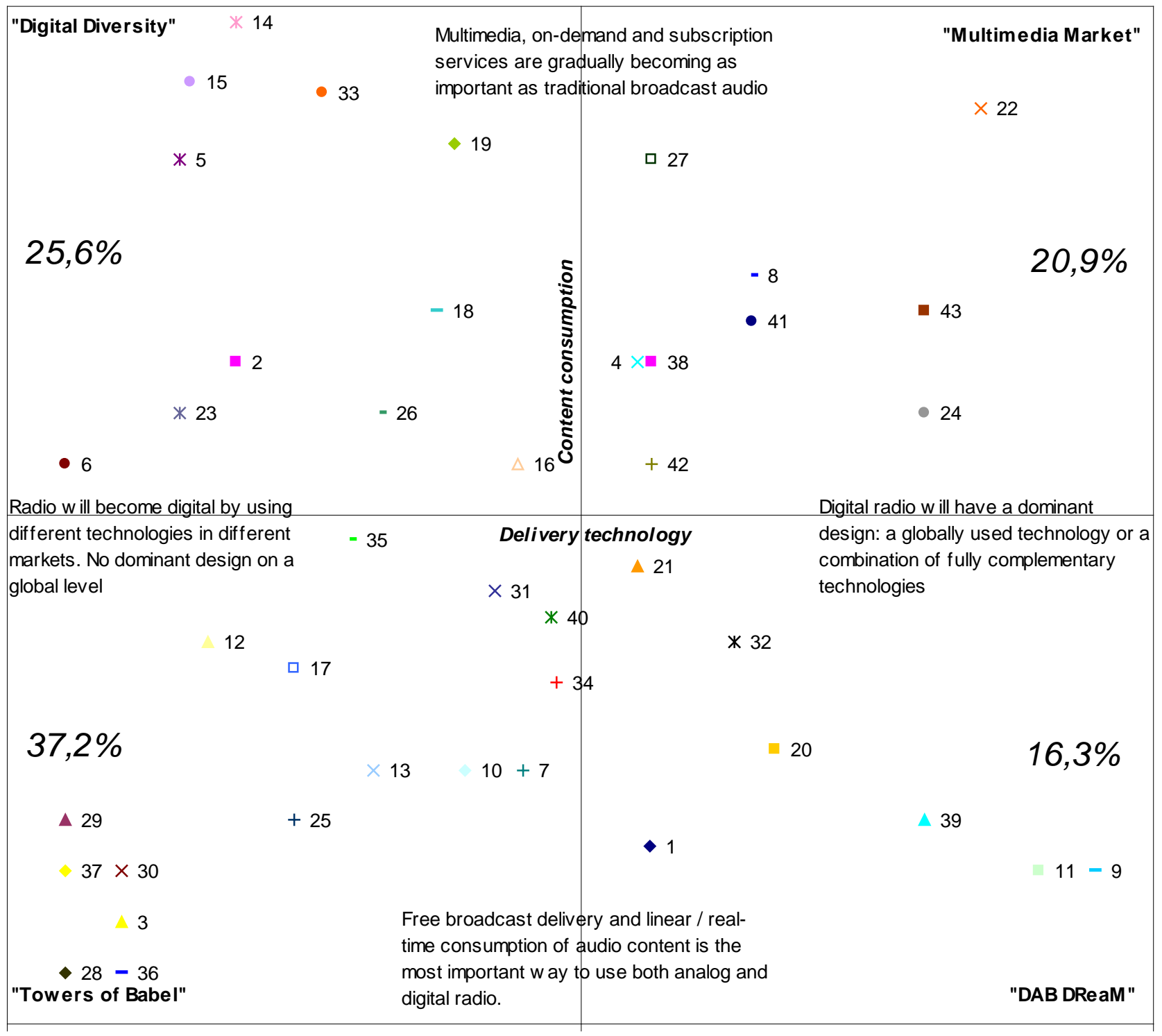


The Future of Radio is Still Digital 33

\section{Notes}

${ }^{1}$ RTE் (Radio Telefís Éireann) launched a new DAB trial in January 2006 and expanded its test services in 2007.

${ }^{2}$ Descriptions and analyses of these different digital audio delivery technologies have been collected to a separate database at the DRACE intranet website (http://www.drace.org). Because of the limitations of this article, all the systems behind the acronyms are introduced here only as brief endnotes.

${ }^{3}$ ISDB-T (Integrated Services Digital Broadcasting -Terrestrial) is a Japanese digital broadcasting system, which has several technical commonalities with European DAB and DVB-T. It was designed to provide a one universal type of system for all terrestrial digital broadcasting, including TV and radio (ISDB-TSB is for sound broadcasting).

${ }^{4}$ IBOC (In-Band, On-Channel) is an American proprietary digital system based on analog radio channel allocations, currently marketed as HD (Hybrid Digital or High Definition) Radio. It is designed to bring digital audio transmissions to the same analog frequencies on which the stations already operate: the digital signal is transmitted on both sides of the analog radio signal.

${ }^{5}$ DRM (Digital Radio Mondiale) was designed in Europe to use the existing AM channel allocations, but it has been further developed also for FM band. Broadcasters using DRM can keep their own transmitters instead of joining a common multiplex like in DAB and DVB-T. On the AM dial, DRM can cover very large areas with only few transmitters.

${ }^{6}$ DVB-T (Digital Video Broadcasting- Terrestrial) was designed in Europe based on DAB experience and it shares the many of the same features with DAB like channel multiplexes. Unlike DAB, DVB-T was not designed primarily for mobile but stable roof-top antenna reception. With its higher data capacity, it suits better for video and multimedia than DAB. DVB-S for satellite broadcasting is based on the same technology.

${ }^{7}$ UMTS (Universal Mobile Telecommunications System) and HSDPA (High Speed Downlink Packet Access) are able to deliver also significant amounts of other data like streaming audio from the Internet to a handset. MBMS (Mobile Broadcast / Multicast Service) means that IP-based multimedia broadcasting services could be offered via already existing GSM and UMTS mobile networks.

${ }^{8}$ DMB (Digital Multimedia Broadcasting) is a multimedia update of the DAB system, which was created in South Korea. It provides good mobile reception like DAB combined with both new audio and video encoding standards, which make DMB not only more versatile but also more efficient. However, DMB does not support Internet Protocol (IP) and that is why also IP-DAB has been developed. DVB-H (Digital Video Broadcasting-Handheld) is a DVBbased mobile system, which links together multimedia broadcasting and mobile phone networks. It delivers digital media using IPDC (Internet Protocol Datacast) system and it has high channel capacity per multiplex. Nokia has been very active to develop the system, but the standard is open. MediaFLO (Media Forward Link Only) is a proprietary technology developed by Qualcomm to bring digital television to mobile phones in the USA. According to Qualcomm, MediaFLO has better performance than any other multimedia broadcasting system.

${ }^{9}$ Besides WLAN (Wireless Local Area Network), there are also larger wireless broadband systems based on other digital technologies like WiMAX (Worldwide Interoperability for Microwave Access), Flash-ODFM (FlashOrthogonal Frequency-Division Multiplexing) and UMTS.

${ }^{10}$ There was never any intention to use the Delphi method (Linstone \& Turoff 1975) for this study as a second round of interviews was not possible due to the limited resources of the project.

${ }^{11}$ Experimental FM IBOC broadcasting was approved in Canada in October 2007.

${ }^{12}$ There are already two groups planning pan-European satellite radio services. WorldSpace is going to start from Italy in 2008, while Ondas Media plans a full European launch in 2010 (BBC 2006).

${ }^{13}$ In January 2007, European Space Agency (ESA) introduced a new satellite multimedia car radio system, which is designed to use already existing, old TV satellites. The system is also designed to work without a terrestrial filler network (ESA 2007, BBC 2007).

${ }^{14}$ An agreement over the Internet streaming music royalties in Finland was finally reached in June 2007.

${ }^{15}$ This kind of multi-standard receivers became available in November 2006 (RadioScape 2006)

${ }^{16}$ In Finland, for example some cities have started to deliver unedited live audio stream from their monthly city council meetings over the Internet (cf. http://www.espoo.fi). You may also listen to live stream from horse races (https://www.fintoto.fi/help/en/introduction/totoradio.htm ) or live classical music concerts (http://www.econcerthouse.com) over the Internet.

${ }^{17}$ World DAB has tried to address these same problems with the adoption of new audio codec and closer cooperation with multiplex-free DRM (Hallet 2005). 